Publ. RIMS, Kyoto Univ.

12 Suppl. (1977), 367-375.

\title{
Old Problems and New Hopes in S-Matrix Theory
}

\author{
by
}

Tullio REGGE*

1) The problem of constructing a convenient ser of axioms for the $S$-matrix directly in terms of the scattering variables and without recourse to conventional field theory is still receiving wide attention by theoretiticians in recent years. The ingredients which go into these attempts are well-known. There is relativistic invariance, unitarity, and finally some degree of analyticity. The hope is that by combining them according to some recipe one obtains the $S$-matrix. The trouble with this program is that really no one seems to know how to combine the ingredients in the proper order; moreover there is wide disagreement as to what analyticity really means. It is on this last point that $I$ wish to concentrate the discussion. Obviously Lorentz invariance and unitarity have quite an ambiguous meaning. Every attempt toward $S$-matrix theory usually starts with the rather ambitious goal of doing away with conventional field theory. The basic philosophy is opposite to that of constructive field theory whose remarkable progress in recent years has overshadowed any alternative approach.

My personal belief however, is that the possibility of different approaches originates from a basic inadequacy of the theory and that ultimately field theory and $S$-matrix theory will be unified. Secondly the constructive approach is still facing tremendous difficulties and is still far removed from any physical application; but basically I feel that committing ourselves to any particular philosophy at this early stage is an essentially unsound strategy.

2) Analyticity statements usually follow from causality through the

Received September 22, 1976.

* Institute for Advanced Study, Princeton, New Jersey 08540.

Research sponsored by the National Science Foundation Grant No. GP-40768X. 
well-oiled machinery of dispersion relations. However this limited kind of analyticity is usually unsatisfactory to the $S$-matrix physicist. For two basic reasons. 1) It bears the clear imprint of the original sin of field theory. 2) It is too limited and it remains meaningful really only at the lowet thresholds. In order to obviate these shortcomings one looks at individual contributions of Feynman relativistic amplitudes (FRA) in the conventional perturbative approach. Here the scattering amplitude appears as the sum of infinitely many FRA of increasing complexity. Each FRA in principle can be completely investigated.

There is no reason however, for the $S$-matrix to be the sum of the FRA and in fact, this is likely not the case. The basic assumption is however that the FRA represents the local behaviour of the amplitude and that the whole picture can be recovered by gluing together all these local behaviours. Each FRA is a multivalued analytic function of the relevant variables whose branching locus is in general an extremely complicated reducible algebraic variety. Luckily the set of singularities is very well defined by the Landau rules. By introducing two scattering amplitudes into the unitarity relation we see that if each of the factors has a given singularity locus then the resulting amplitude must be singular on a variety defined by some algebraic reduction procedure from those of the factors. In other words unitarity tells us that given a Landau variety we can form a third one through unitarity. The set of all singularities must be closed under this composition rule. One sees quickly that starting from the simplest polar singularities at the lowest perturbative orders the set is indefinitely enlarged and includes eventually all Landau singularities of every permissible FRA. This set is probably denumerably infinite and the algebraic degree of the variety grows exponentially with the perturbative order.

At this point one needs to gain some control on these proliferating singularities. The main tool is the pleasant discovery that the physical amplitude is singular only on a very limited real subset of the whole variety (the so-called alpha positive part) and that this subset has rather nice support properties. In fact, by increasing the order of the FRA the subset tends to move away into high energy regions.

One has then two basic options for the resulting strategy. The first 
is to restrict the investigation of the FRA and of the $S$-matrix to the real analytic domain. The second is to jump into the complex domain. The first approach has received a strong boost from the remarkable algorithm of the hyperfunctions and related microlocal calculus developed by Sato and co-workers. This method seems to fit very nicely with the general approach developed by Stapp, Iagolnitzer and others. In particular the concent of MOS seems to be a natural gluing tool in fitting together the singular behaviour of the partial FRA. The second strategy has been put into practice on the $S$-matrix by the Cambridge group (Olive, Eden, Polkinghorne, Landshoff et. al.) in a rather unsystematic fashion because of the tremendous difficulties involved. A related approach by Pham and Fotiadi, Lascoux and Froissart has used the machinery of algebraic topology on individual FRA. Finally, in a set of related papers Ponzano, Speer, Westwater and myself (PSWR) have developed an algebraic approach on FRA which works on selected classes of FRA (single loop graphs, 2-vertex self-energy graphs, necklace graphs). None of these last methods has been applied to the $S$-matrix itself.

What are the relative virtues of the two strategies? As far as I can see, the real analytic approach has the merit of working with quantities having a direct physical meaning and direct physical intuition is certainly of great help. Also the novel mathematical techniques of the Japanese school hold great promise of the future development. On the negative side I would place the still pending problem of classifying all the Landau varieties. The position of these depends on the masses of the interacting particles. By varying these the relative ordering of the singular loci changes often in an unpredictable manner. The occurrence of cusps and of accidental degeneracies follows from a set of complicated inequalities among masses which appear difficult to control in an ambitious global theory, but perhaps manageable in a local context.

In the complex domain the problem of controlling the Landau curve is less crucial. There is however an enormously larger freedom of carrying out analytic continuations. The scattering amplitude (or at this stage more modestly an individual FRA) is initially defined in a suitable neighborhood and then continued outside. The resulting function turns out to be an element of a Nillson class and the set of all possible analytic 
continuation is identified with the fundamental group of the compliment of the Landau varities. By restricting the theory to a thin slab around the real domain it is possible to translate part of the results and concepts of the complex approach into those of the real analytic approach. There are in fact, differential equations in the complex domain for the FRA which are strongly related to the MOS proposed by the Sato group. The drawback is, of course, the lack of direct physical interpretation of the amplitudes and the still unsolved problem of formalizing unitarity in the complex domain.

3) The non-Landau singularities. A common difficulty lying ahead in both the complex and real analytic approach is however the occurrence of non-Landau singularities. As we have stated the set of all Landau singularities is actually infinite. We cannot exclude that it may be everywhere dense in the complex domain or perhaps even in the real domain. The study of individual FRA has shown that there are other singularity loci which were not included in the original Landau treatment and do not seem to have a simple physical meaning. These singularities are perhaps best understood by looking at unitarity relations. Generally the cycle of integration in a unitarity integral is compact. It may happen however that for particular values of the physical variable the cycle becomes non-compact and that a "pinch" occurs at infinity with the appearance of a new singularity. In a FRA this is not a disaster for the resuting singularity is tractable with the same techniques as for the standard ones, the relevant exception being the non-validity of the Cutkosky rules. In a $S$-matrix theory the points at infinity are really the accumulation of an indefinite sequence of ordinary singularities, all of which are "pinched" with the integration cycle thus producing an infinite set of Landau singularities accumulating around the non-Landau one. In fact a non-Landau singularity is just the accumulation of infinitely many ordinary ones. The process can be repeated and iterated and the resulting structure becomes hopelessly complicated. A non-Landau locus is then, by abuse of language, just as far away as infinity. In a FRA they appear simpler because all accumulating Landau singularities are collapsed into a single locus. In a way in this case the non-Landau locus is simply 
a way to describe the effect of all the singularities of the other graphs. I feel that this basic difficulty will appear in both approaches.

4) A more basic question concerns the meaning and the kind of information one hopes to extract from an $S$-matrix theory. In the ideal case one would like to estabilish a set of axioms which uniquely characterize the scattering amplitudes and stable states of the theory.

In all applications of the theory however, the most significant results have correlated the amplitudes for relatively simple scattering processes to the masses of a few elementary particles. Is the theory supposed to give, it its final form, also the masses of all particles and not simply to correlate them to other interesting predictions? In other words, are there infinitely many parameters left into the theoretical frame, which need to be specified before we attempt comparison with experiment? In the early views of Chew this was not the case but it may happen that a physical theory merely predicts a manifold of possible physical worlds, and that the one we live in is chosen because it is the only one allowing biochemistry.

In this view the theory would merely provide us with a set of relations between masses, phase shifts, binding energies and the like without specifying all of them. Further axioms, not germane to conventional $S$-matrix theory, would be needed in order to establish uniqueness of the predictions.

5) The construction of an $S$-matrix is enormously complicated by the multi-sheeted structure of the amplitudes and by the intricated nature of the singular set. In the PRSW approach the main hope was that the singular set and the monodromy group could be calculated directly from the FRA and that a convenient generalization of Hibert 21st problem would provide the natural frame for an axiomatic approach to the $S$ matrix.

The program has faced the following, still unresolved difficulties.

A) The singular locus, the fundamental group of its complement and the monodromy group can be calculated only for very restricted classes of FRA. For instance multi-loops diagrams generally are out of 
reach.

B) Although FRA characterize the local behaviour of the $S$-Matrix no attempt was made to cast this correspondence in a more systematic form. In particular, the fundamental group of the complement of the set of all Landau singularities can be defined probably only through a limiting procedure (projective limit) on the set of infinite discrete groups, which is not free of dangers. This comment applies even more on monodromy group.

C) Even granting the complete construction of the fundamental and monodromy groups the proper setting and even the existence of a solution of the 21st Hilbert problem has not been proved for unions of inifnitely many algebraic varieties.

D) Finally, one needs to specify the local Laurent expansion near the singular locus and not only the monodromy group. In a sense therefore problem is not the one proposed by Hilbert but rather the type of investigation carried out in particular cases by Lappo-Danilevsky, Schlesinger and others.

It has to be noted however, that the fundamental group is just a very neat and convenient way to classify all Riemann sheets of multivalued function and that will appear in a natural way whenever this task is attempted. In particular, even the real analytic approach will have to face the need for a systematic treatment of the multi-sheeted structure of the amplitudes and will encounter the fundamental group in a more or less disguised form. Also, quite remarkably, although the analysis in PRSW was ostensibly done in the complex domain, the results really could have been obtained by restricting it to a neighourhood of the real domain with no relevant changes.

For this reason I feel that there is no real incompatibility between the real analytic and the complex approach.

6) I shall recall in this section a few definitions and relevant ideas of the complex analysis. In dealing with a given FRA there is no restriction in considering it as a Nillson function $A(z)$ defined over $C^{n}-L^{n-1}$ or possibly $\boldsymbol{C} \boldsymbol{P}^{n}-L^{n-1}$, where $L^{n-1}$ is the singular locus consisting of the union of a large, but finite number of irreducible algebraic varieties. 
The physical amplitude is initially defined in a neighbourhood $U$ of a base point $P$, not intersecting $L$. Suppose now that the following conditions are satisfied:

a) $A(z)$ can be extended to a holomorphic function over the universal covering space of $C^{n}-L^{n+1}$. This means that $A$ can be continued along any element $g$ of $\pi_{1}\left(C^{n}-L^{n-1}\right)$ yielding an analytic continuation $A_{g}$ defined in $U$.

b) There are growth conditions implying that the extended $A$ is bounded by a polynomtal in the neighbourhood of $L$.

c) All $A_{g}$ are linear combinations, with constant coefficients $\lambda_{i}(g)$ of a basic set of amplitudes $A_{i}$ within $U$ :

$$
A_{g}=\sum_{i=1}^{N} \lambda_{i}(g) A_{i}
$$

By acting with an element of $\pi_{1}$ on $A_{i}$ we find therefore that

$$
\left(g A_{i}\right)(z)=\sum_{k=1}^{N} \mu_{k i}(g) A_{k}(z) \quad i=1, \cdots, N
$$

The matrix $\mu_{k i}(g)$ is readily seen to provide a linear representation of $\pi$, which is by definition the monodromy group $G$.

The general results in this field imply that if $G$ is given then $A$ is specified only as follows:

$$
A(z)=\sum_{e=1}^{N} R_{e}(z) A^{e}
$$

where the $R_{e}$ are generic rational functions and the $A^{e}$ form a set of $N$ independent (over the field of rational functions) solutions of the problem. The derivative $\frac{d A^{e}}{d z}$ with respect to any variable $z$ has still the same monodromy group, therefore we must have a differential equation of the type:

$$
\frac{d A^{e}}{d z}=\sum_{k} R_{k}^{e} A^{k}
$$

On each amplitude $A$ and course for $A$ itself implies a single differential equation most obviously be related maximally overdetermined systems (MOS) in Sato's approach. Conversely the existence of such a system implies the Nillson property for its solution. There is no easy way to 
deduce the monodromy group from the differential equation, and vice-versa that I know of besides solving the equations themselves in terms of known functions, if possible.

From the brilliant analysis of the Japanese school the MOS for a given FRA is essentially known from the topology of the graph. The MOS pseudo differential operator however, corresponds roughly to the leading order of the conventional complex differential equations and we have no equally powerful algorithm for the constriction of the complete system. This is the main reason why in PRSW the effort was directed toward the construction of the monodromy group which appears to be more tractable. Clearly the differential equation plus boundary conditions in the form of $N$ constants given at $P$ determines completely the solution and therefore a specific amplitude. Differential equations on one side and monodromy group plus growth conditions on the other contain the same information packed in a radically different way and the connection between the two should be further elucidated before any attempts on the $S$-matrix are carried out in this fashion.

7) A last difficulty arises from the presence of infinitely many Landau singularities. This difficulty is common to all approaches. Using Sato conjecture one has to consider pseudodifferential operators of infinite order. This is not a novelty in the theory. What is a novelty is perhaps the occurrence of second kind singularities with associated accumulation of conventional Landau singularities which will require an ad hoc supplementary analysis.

Perhaps the best strategy is to give up entirely the idea of defining $S$-matrix amplitudes globally with a single equation of a single integral. The axiomatic field theory of Haag and Araki tries to reach a definition of the complete algebra of observables by nesting together local $C^{*}$ algebras defined on open sets of space-time in a manner consistent with invariance and causality. Similarly either MOS or (in the complex) monodromy groups and associated structures should be defined only on selected classes of subsets in momentum space which are then glued together with appropriate axioms of identification. In this sense one has a set of privileged subsets of the space of scattering variables for which the monodromy 
group, the fundamental group and when the case, MOS are defined in the usual fashion. Sets of this sort need a definition of "compactness" in order to avoid an actual infinity of singular algebraic varieties with intractable pathologies. It is not difficult to introduce this notion of compactness. A set is "compact" if the amplitude satisfies a MOS of finite order in it or (in the complex) if the amplitude continues into a Nillson function with $N$ finite and a finite number of Landau singularities (and therefore no non-Landau singularity).

The difficult part is to create an algorithm out of this definition. The intersection of compact sets is itself compact but the union is not necessarily so and this limits greatly the usefulness of the definition. Finally, there is always the last hurdle on how to treat unitarity efficiently in terms of these linear structures. In spite of the tremendous difficulties lying ahead I fell confident that the $S$-matrix theory is far from dead and that much is to be learned from the new mathematical methods and in turn much new interesting mathematics will be created by attempting to formalize it.

\section{Acknowledgements}

The author is deeply indebted to Prof. Sato for the hospitality at RIMS and the invitation to join the Oji International Seminar on Algebraic Analysis. The author also thanks Dr. Kawai, Dr. Stapp, Dr. Westwater and of course, Dr. Sato for many interesting discussions.

\section{References}

[1] Regge, T., Actes du Congres International des Methematiciens, 1/10 Septembre 1970/Nice, France, p. 9.

[2] Ponzano, G., Regge, T., Speer, E. R. and Westwater, M. J., The monodromy rings of a class of self-energy graphs, Comm. Math. Phys. 15 (1969), 83-132.

[3] Regge, T., "The fundamental group of Poincare and the Analytic properties of Feynman relativistic amplitudes", Nobèl Symposium 8, Elementary Particle Theory, ed., Nils Svartholm, N. Y., Interscience, 1969.

[4] Ponzano, G., Regge, T., Speer, E. R. and Westwater, M. J., The monodromy rings of one loop Feynman integrals, Comm. Math. Phys. 18 (1970), 1-64. 
